\title{
Feasibility of Predicting MCI/AD Using Neuropsychological Tests and Serum $\beta$-Amyloid
}

\author{
Cheryl A. Luis, Laila Abdullah, Ghania Ait-Ghezala, Benoit Mouzon, Andrew P. Keegan, \\ Fiona Crawford, and Michael Mullan
}

Roskamp Institute, 2040 Whitfield Avenue, Sarasota, FL 34243, USA

Correspondence should be addressed to Cheryl A. Luis, cluis@rfdn.org

Received 13 October 2010; Revised 23 March 2011; Accepted 27 March 2011

Academic Editor: Katsuya Urakami

Copyright (c) 2011 Cheryl A. Luis et al. This is an open access article distributed under the Creative Commons Attribution License, which permits unrestricted use, distribution, and reproduction in any medium, provided the original work is properly cited.

\begin{abstract}
We examined the usefulness of brief neuropsychological tests and serum $\mathrm{A} \beta$ as a predictive test for detecting $\mathrm{MCI} / \mathrm{AD}$ in older adults. Serum A $\beta$ levels were measured from 208 subjects who were cognitively normal at enrollment and blood draw. Twentyeight of the subjects subsequently developed MCI $(n=18)$ or $\mathrm{AD}(n=10)$ over the follow-up period. Baseline measures of global cognition, memory, language fluency, and serum $A \beta_{1-42}$ and the ratio of serum $A \beta_{1-42} / A \beta_{1-40}$ were significant predictors for future MCI/AD using Cox regression with demographic variables, APOE $\varepsilon 4$, vascular risk factors, and specific medication as covariates. An optimal sensitivity of $85.2 \%$ and specificity of $86.5 \%$ for predicting MCI/AD was achieved using ROC analyses. Brief neuropsychological tests and measurements of $A \beta_{1-42}$ obtained via blood warrants further study as a practical and cost effective method for wide-scale screening for identifying older adults who may be at-risk for pathological cognitive decline.
\end{abstract}

\section{Introduction}

An exponential rise in Alzheimer's disease $(\mathrm{AD})$ prevalence rates is predicted to parallel the aging of baby boomers creating a potentially unsustainable economic burden to the healthcare system. Delaying the onset or progression of $\mathrm{AD}$, even modestly, by earlier pharmacological intervention could substantially reduce the economic and psychosocial impact of the illness $[1,2]$. Unfortunately, many AD patients remain undiagnosed or go undetected until the later stages of disease. Insights into the underlying pathological mechanisms involving beta-amyloid plaque deposition within the brain have led to the development of a host of antiamyloid agents [3] that are in various stages of clinical investigation. There is now a scientific consensus that the pathological events in $\mathrm{AD}$ initiate decades before clinical symptoms become apparent, and if disease modification is realized in the coming decades, the need for improved methods of early detection prior to the overt clinical signs will be accentuated.

Traditionally, neuropsychological measures, particularly those that tap cognitive abilities subsumed by the hippocampal formation such as episodic memory, have shown usefulness in identifying cognitively normal elders who subsequently develop $\mathrm{AD}[4,5]$. Decrements in semantic memory and concept formation have been shown to occur nearly a decade before the development of AD [6]. Performance on visual-spatial and verbal memory measures in midlife have also been shown to predict later memory loss [7]. Neuropsychological measures are noninvasive and generally cost effective. However, individuals with very high premorbid intellectual abilities experiencing incipient cognitive decline may go undetected, and false positives are possible in individuals with a low level of intellectual abilities. Also appropriate interpretation of extensive neuropsychological testing requires a high degree of expertise and training, which limits its use in routine clinical settings.

The advancement of molecular imaging tracers that bind to amyloid, such as Pittsburgh Compound B (PIB) or longerlived probes (e.g., FDDNP), offers a non-invasive in vivo method to detect and quantify brain amyloid deposition $[8,9]$. However, this approach for presymptomatic detection is economically impractical for routine use given the current costs and restrictions on "medically necessary" use. Similarly, biomarkers including $\mathrm{A} \beta_{1-42}$ and phosphorylated tau (also 
implicated in AD pathology) in cerebral spinal fluid (CSF) can predict subsequent cognitive decline $[10,11]$, but lumbar puncture carries risks and is inconvenient for wide-scale use in cognitively impaired elderly subjects.

Blood-based biomarkers have more practical applicability for routine use and are likely to be more cost effective than both CSF and imaging procedures. Consequently, measurement of $A \beta_{1-40}$ and $A \beta_{1-42}$ in blood is increasingly being explored and shows potential in identifying individuals at the preclinical stage of $\mathrm{AD}$ [12-14]. It has been reported that CSF $A \beta$ levels are subject to high diurnal fluctuations with extremely high variability reported over 12 hours [15]. Over days and weeks, $A \beta$ in blood appears more stable than CSF [16-18]. Furthermore, serum contains more $A \beta$ than plasma [16], possibly due to the release of bound $A \beta$ during the clotting process [19]. Hence, serum $A \beta$ appears suitable for use in predicting MCI/AD and optimal sensitivity, and specificity is probably achievable if combined with current diagnostic procedures, such as brief neuropsychological testing.

In this study, we examined the usefulness of brief neuropsychological tests in combination with blood $\mathrm{A} \beta_{1-40}$ and $A \beta_{1-42}$ as a predictive test for detecting MCI/AD in at-risk older adults at a pre-symptomatic stage. Such an approach will be more practical for clinical use and be germane in designing large-scale prevention trials.

\section{Methods}

2.1. Subjects. Participants included a subset of subjects enrolled in the Alzheimer's Disease Anti-Inflammatory Prevention Trial (ADAPT). ADAPT was a randomized, placebo-controlled, multicenter primary prevention trial sponsored by the National Institute on Aging. The Roskamp Institute served as one of six recruitment sites located across the US. Subjects were randomly assigned to one of three groups: celecoxib (200 $\mathrm{mg}$ b.i.d.), naproxen sodium (220 mg b.i.d.), or placebo. The primary outcome measure of ADAPT was development of AD. Full details of data collection, measurements, and study procedures are available at http://www.jhucct.com/adapt/manall43.pdf and described elsewhere [20].

The inclusion criteria for ADAPT subjects were age of 70 or older at enrollment, a self-reported family history of ADlike dementia, and normal cognitive performance on a brief battery of neuropsychological tests. Recruitment for ADAPT began in 2002, and the study was completed in 2007. In 2005, the Roskamp Institute initiated a proteomic ancillary study (F. Crawford, PI) involving blood draw from these subjects. The inclusion criteria for this ancillary study stipulated that each subject was an active ADAPT participant and had met all the ADAPT inclusion and exclusion criteria. An approval was obtained from the ADAPT Steering Committee and a centralized IRB. A separate consent was also obtained from each subject who participated in the ancillary study.

Two hundred and fifteen subjects from the Roskamp ADAPT cohort enrolled in the proteomic ancillary study. At the time of blood draw, subjects maintained cognitively normal status as determined by their performance on an annual cognitive assessment battery. These assessments continued for an additional two years following the blood draw. Blood was collected during the semi-annual followup visits, and the cognitive assessments were performed at the baseline visit and at the annual visits. The time from baseline cognitive testing to the diagnosis of MCI/AD was 4.06 years $( \pm 1.3 \mathrm{SD})$. Timeframe from baseline cognitive testing to blood draw was 2.25 years $( \pm 0.71 \mathrm{SD})$ and from blood draw to diagnosis was 1.79 years $( \pm 1.2 \mathrm{SD})$. The cognitive measures completed at baseline and annual followup included the Modified MiniMental State Examination (3MS) [21]; the Hopkins Verbal Learning Test-Revised (HVLT-R) [22]; Digit Span (forward and backward) from the Wechsler Adult Intelligence ScaleRevised (WAIS-R) [23]; a Generative Verbal Fluency test (supermarket items); the narratives from the Rivermead Behavioral Memory Test (RBMT) [24]; the Brief Visuospatial Memory Test-Revised (BVMT-R) [25]. The Mini-Mental State Examination (MMSE) [26] was extracted from 3MS. Alternate forms were utilized annually for the HVLT-R, RBMT, and BVMT-R on each subsequent annual visit. Subjects also completed the 30-item Geriatric Depression Scale [27] and a self-rating scale of memory functions [28]. Collateral respondents completed the Dementia Severity Rating Scale (DSRS) [29]. Due to significant intercorrelations between these tests, analyses described below are limited to those baseline cognitive tests that were sensitive to early changes (i.e., verbal learning and memory) associated with $\mathrm{MCI} / \mathrm{AD}$ [30] or tests that were similar to those previously shown to be associated with $A \beta$ levels [31].

Normative data from the Cache County study was used to develop the standardized cut-off scores utilized in ADAPT [32]. Individuals who scored below the cut scores on annual cognitive assessments underwent further dementia workup including physical and neurological examinations, laboratory studies (i.e., $\mathrm{CBC}$, chemistry count, sedimentation rate, vitamin $\mathrm{B}_{12}$ and folic acid levels, thyroid test, and syphilis serological test), and neuroimaging (i.e., MRI or CT), as applicable. A more comprehensive neuropsychological assessment was also administered by a neuropsychologist as part of the dementia work-up. This battery of tests consisted of the expanded Consortium to Establish a Registry for Alzheimer's Disease (CERAD) battery [33]; Logical Memory I and II of the Wechsler Memory Scale-Revised [34]; Benton Visual Retention Test [35] (Benton); a generative fluency test (animals); Control Oral Word Association Test (COWAT; CFL) [36]; The Trail Making Test [37]; Symbol Digit Modalities Test (SMDT) [38]; Shipley Vocabulary [39].

Following completion of all components of the dementia work-up, a consensus team determined cognitive status using published diagnostic criteria. The annual battery was not utilized in diagnostic determination. The diagnosis of $\mathrm{AD}$ was made using NINCDS-ADRDA [40] and amnestic mild cognitive impairment (MCI) using Petersen criteria [41]. All MCI patients were considered to be amnestic MCI, as they only had memory impairment, but maintained normal activities of daily living and overall had a wellpreserved cognition in other cognitive domains. Ample 
evidence indicates that amnestic MCI patients may be in a transitional stage between normal aging and $\mathrm{AD}$ with $85 \%$ of these subjects converting to $\mathrm{AD}$ over a 7-year period [42]. Additional evidence comes from an imaging study which demonstrated that the pattern of brain atrophy in amnestic MCI patients is typical of that observed in AD patients [43]. It is then reasonable to combine these diagnoses in a single category, thus allowing a large enough numbers to supply statistical power. Of the 215 subjects who gave blood for the ancillary study, two developed non-AD dementia, and another subject died with cognitive status unknown. Blood $\mathrm{A} \beta$ values were unavailable for 4 subjects. Of the remaining subject pool of 208 used in these analyses, 28 subjects met criteria for either $\mathrm{AD}(n=10)$ or $\mathrm{MCI}(n=18)$ in the two years following blood draw.

2.2. Sample Collection, Preparation, and Measurements. Blood draws for $\mathrm{A} \beta$ measurement and APOE genotyping were conducted by trained phlebotomists. Serum from blood was prepared and processed using standard laboratory procedures [16]. The serum $A \beta$ content was determined, as per manufacturer's instructions, using the ELISA kits for human $\mathrm{A} \beta_{1-40}$ and $\mathrm{A} \beta_{1-42}$ and the inter-assay $\mathrm{CV}$, and the intraassay CV was reported to be $\leq 10 \%$ (Invitrogen, Calif). Additional details are provided elsewhere [16]. DNA was extracted from whole blood for APOE genotyping using Pure Gene Kits (Gentra systems, Calif), and APOE genotyping was performed using previously established methods, as described elsewhere [16]. APOE genotypes were unavailable for 4 individuals, but these were included in the analyses.

2.3. Statistical Analyses. The data set was range checked, and prior to analyses, the dependent and independent variables were examined for missing data, outliers, and violations of the normalcy assumption. Differences among groups on demographic variables, neuropsychological variables, and serum $\mathrm{A} \beta_{1-40}$ levels were examined using either the student's $t$-test or $\chi^{2}$ analyses, depending on the type of variable measurement. The Mann-Whitney test was employed if parametric assumptions were not met.

Time-updated Cox regression modeling was used to test whether neuropsychological test scores, $A \beta$, or a combination of both can predict conversion to MCI/AD in individuals who were cognitively normal at baseline. Potential confounding variables shown to impact risk for cognitive decline included age, education, gender, APOE status, serum creatinine, triglycerides, presence of APOE $\varepsilon 4$ allele, and history of vascular disease as determined by treatment with statins or antihypertensive medication which were entered as covariates. The latter variables, coded dichotomously, have been previously shown to impact $A \beta$ levels [44]. Because previous analyses revealed a nonsignificant increase of $\mathrm{AD}$ risk with naproxen in this cohort [45], we also controlled for this effect.

Logistic regression modeling was employed to construct receiver operator curves (ROC) to examine the predictive performance of neuropsychological measures from the baseline visit and serum $A \beta$ levels in diagnoses of MCI/AD.
ROC curve comparisons were based on area under the curve (AUC), SE, and the associated 95\% confidence interval (CI). We subsequently calculated sensitivity of the various models using the predicted probability of each subject by logistic regression modeling with specificity of at least eighty percent. Post hoc power calculations using the G-power software for multivariate regression analyses utilized here suggest a power of nearly $100 \%$ at the alpha value 0.05 for the current sample size, total number of predictors, and the observed effect size. All analyses were conducted using the SPSS version 16.0 for Macintosh.

\section{Results}

The mean age and education of the sample was 76.7 (SD = $3.9)$ and $14.6(\mathrm{SD}=2.8)$ years, respectively. The majority of the sample was Caucasian (98.1\%), and 51.9\% were male. Despite the cohort's self-report of enriched family history, less than one-third of the total sample $(31.7 \%)$ carried at least one APOE $\varepsilon 4$ allele, a frequency similar to the general population [44]. Comparisons on variables between subjects who remained cognitively normal and those who declined over the short follow-up period are reported in Table 1. Although all subjects at enrollment performed within the normal limits based on the established cut-off scores, those that ultimately declined had generally poorer scores on the 3MS, MMSE, and all memory measures. The two groups were also significantly different on serum $\mathrm{A} \beta_{1-42}$ levels and $\mathrm{A} \beta_{1-42} / \mathrm{A} \beta_{1-40}$ ratios prior to diagnoses of MCI/AD. Only $23 \%$ of the cognitively normal individuals had serum $\mathrm{A} \beta_{1-42}$ in the lowest quartile compared to the nearly $50 \%$ of the diagnostic group (44\% of MCI subjects and $50 \%$ of $\mathrm{AD}$ subjects).

Time-dependent Cox regression analyses were performed to examine the relationship between these cognitive tests and $\mathrm{A} \beta$ on the prediction of subsequent conversion to MCI/AD. All neuropsychological analyses were adjusted for age, gender, and education, but no adjustment for the study medications was required as these were baseline scores. Cox regression analyses show that the model using neuropsychological tests predicted MCI/AD ( -2 log-likelihood $\left.=206.51, \chi^{2}=52.11, \mathrm{df}=8, P<.001\right)$. Significant individual neuropsychological measures were $3 \mathrm{MS}(\beta=-0.25 \pm 0.06$, Wald $=17.78, P<.001)$; generative verbal fluency $(\beta=$ $0.12 \pm 0.04$, Wald $=8.09, P<.004)$; HVLT-R scores $(\beta=$ $0.24 \pm 0.11$, Wald $=4.58 P<.032$ ).

Cox regression analysis showed that $\mathrm{A} \beta_{1-42}$ measured in the lowest two quartiles compared to the highest quartile was a significant individual predictor of conversion to $\mathrm{MCI} / \mathrm{AD}$ in this model $\left(-2 \log\right.$-likelihood $=197.47, \chi^{2}=38.41$, $\mathrm{df}=15, P<.001)$. The regression analysis utilizing the $\mathrm{A} \beta_{1-42} / \mathrm{A} \beta_{1-40}$ ratio found similarly significant results $(-2$ log-likelihood $=204.69, \chi^{2}=36.10, \mathrm{df}=14, P<.001$ ) with the lowest ratios being most predictive of subsequent conversion to MCI/AD. The final full model, adjusting for confound and the study medications, included HVLT-R, fluency, $3 \mathrm{MS}, \mathrm{A} \beta_{1-42}$ levels, and $\mathrm{A} \beta_{1-42}$ quartiles $(-2$ loglikelihood $\left.=166.25, \chi^{2}=74.55, \mathrm{df}=18, P<.001\right)$ with 
TABLE 1: Variable comparisons between groups.

\begin{tabular}{|c|c|c|}
\hline Variable & $\mathrm{MCI} / \mathrm{AD}(n=28)$ & Controls $(n=190)$ \\
\hline Age & $77.8 \pm(3.9)$ years & $76.6 \pm(3.9)$ years \\
\hline Education & $14.61 \pm(3.2)$ years & $14.63 \pm(2.8)$ years \\
\hline$\%$ Male & $67.9 \%$ & $49.4 \%$ \\
\hline$\%$ APOE $\varepsilon 4$ carrier & $42.3 \%$ & $32.4 \%$ \\
\hline & \multicolumn{2}{|c|}{ Means \pm SD } \\
\hline $3 \mathrm{MS}$ & $92.93 \pm(4.0)^{*}$ & $96.7 \pm(3.0)$ \\
\hline MMSE & $28.29 \pm(2.1)^{*}$ & $28.98 \pm(1.3)$ \\
\hline HVLT-R & $8.11 \pm(2.1)^{*}$ & $9.85 \pm(2.0)$ \\
\hline \multicolumn{3}{|l|}{ Digit Span: } \\
\hline Forward Score & $8.36 \pm(2.3)$ & $8.27 \pm(2.0)$ \\
\hline Backward Score & $6.93 \pm(2.1)$ & $6.87 \pm(1.9)$ \\
\hline Generative Fluency & $24.86 \pm(5.8)$ & $25.66 \pm(6.2)$ \\
\hline RBMT & $57.14(25.4)^{*}$ & $75.00 \pm(31.2)$ \\
\hline BVMT-R & $6.46 \pm(2.6)^{*}$ & $8.07 \pm(2.4)$ \\
\hline \multirow[t]{2}{*}{$\mathrm{A} \beta_{1-40}$} & $138.08 \pm(43.72)$ & $146.24 \pm(55.37)$ \\
\hline & \multicolumn{2}{|c|}{ Median (25th, 75th quartile) } \\
\hline $\mathrm{A} \beta_{1-42}$ & $7.23(1.97,17.49)^{* *}$ & $12.38(6.28,23.20)$ \\
\hline $\mathrm{A} \beta_{1-42} / \mathrm{A} \beta_{1-40}$ ratio & $0.05(0.02,0.10)^{* *}$ & $0.09(0.05,0.15)$ \\
\hline
\end{tabular}

${ }^{*} t$-Test $P<.05$.

** Mann-Whittney $\mathrm{U} P<.05$.

Note: 3MS = Modified Mini-Mental State Examination; MMSE = Mini-Mental State Examination; HVLT-R = Hopkin's Verbal Learning Test-Revised, Trial 4; RBMT $=$ Rivermead Behavioral Memory Test; BVMT-R = Benton Visual Memory Test-Revised .

fluency, $3 \mathrm{MS}$, and $\mathrm{A} \beta_{1-42}$ in the lowest two quartiles as significant individual predictors of $\mathrm{MCI} / \mathrm{AD}$ in the model. $\mathrm{A} \beta_{1-40}$ was not a significant individual predictor. Similar results were observed when $A \beta_{1-40}$ levels and $A \beta_{1-42}$ quartiles were substituted in this model with $\mathrm{A} \beta_{1-42} / \mathrm{A} \beta_{1-40}$ ratios ( $-2 \log$ likelihood $\left.=168.49, \chi^{2}=72.90, \mathrm{df}=17, P<.001\right)$.

Baseline values for the 3MS, HVLT-R, and generative verbal fluency scores were subtracted from those obtained at the 12-month repeat testing to determine if changes in these measures differ by $A \beta_{1-42}$ and $A \beta_{1-42} / A \beta_{1-40}$ ratios. In unadjusted analyses, among subjects who converted to MCI/AD, the greatest decline for HVLT-R was observed among individuals with the lowest quartile of $\mathrm{A} \beta_{1-42}(-1.17, \pm 2.33 \mathrm{SD})$ and $\mathrm{A} \beta_{1-42} / \mathrm{A} \beta_{1-40}$ ratios $(-0.75, \pm 2.63 \mathrm{SD})$ where individuals in the highest quartile of $\mathrm{A} \beta_{1-42}(1.33, \pm 1.86 \mathrm{SD})$ and $\mathrm{A} \beta_{1-42} / \mathrm{A} \beta_{1-40}$ ratios improved by nearly one point $(0.6 \pm$ $1.82 \mathrm{SD})$. However, these differences were not statistically significant $(P>.05)$. For the $3 \mathrm{MS}$ scores, among subjects who converted to $\mathrm{MCI} / \mathrm{AD}$, those with $\mathrm{A} \beta_{1-42}$ in the lowest quartile declined $(-1.83 \pm 1.28 \mathrm{SD})$ as compared to the highest quartile $(4.83 \pm 1.35 \mathrm{SD})$, and this difference was statistically significant $(\mathrm{F}=3.42, P=.033)$. For $\mathrm{MCI} / \mathrm{AD}$ subjects with the lowest quartile of the $\mathrm{A} \beta_{1-42} / \mathrm{A} \beta_{1-40}$ ratios, the $3 \mathrm{MS}$ values remained ultimately unchanged $(0.16 \pm$ $1.20 \mathrm{SD}$ ), while the scores improved among those with the highest quartile of the $\mathrm{A} \beta_{1-42} / \mathrm{A} \beta_{1-40}$ ratios $(4.33 \pm 1.20 \mathrm{SD})$, and these differences were also statistically significant $(\mathrm{F}=$ 3.10, $P=.046$ ). For generative verbal fluency test, a decline was noted in both the lowest quartile $(-4.17 \pm 1.40 \mathrm{SD})$ and the highest quartile $(-1.17 \pm 2.13 \mathrm{SD})$ of $\mathrm{A} \beta_{1-42}$, and these differences were marginally significant $(F=2.63$,
$P=.073)$. For $\mathrm{A} \beta_{1-42} / \mathrm{A} \beta_{1-40}$ ratios, a similar pattern was observed, but this difference was not statistically significant. Among individuals who remained cognitively normal, while a similar pattern was observed, those with lowest quartile of $\mathrm{A} \beta_{1-42}$ and $\mathrm{A} \beta_{1-42} / \mathrm{A} \beta_{1-40}$ ratios had a larger decline than those with the highest quartile for each HVLT-R $(-0.28 \pm$ $0.27 \mathrm{SD}$ versus. $0.14 \pm 0.33 \mathrm{SD}$, respectively.) and $3 \mathrm{MS}$ $(-1.02 \pm 0.51 \mathrm{SD}$ versus $-0.39 \pm 0.44 \mathrm{SD})$. However, due to the small magnitude of the change in these scores, these differences were not statistically significant. No such change was observed for the generative verbal fluency test (data not shown).

Examination of sensitivity and specificity using ROC analysis revealed the AUC for neuropsychological testing with age, education, and gender as covariates was 0.83 (95\% CI $[0.75-0.91], P<.001$ ). For $\mathrm{A} \beta_{1-42}$ (adjusted for presence of APOE $\varepsilon 4$ allele, vascular risk factors, and associated medications), the AUC was 0.79 (95\% CI [0.70-0.88], $P<$ $.001)$. When neuropsychological testing (3MS, HVLT-R, and Generative Verbal Fluency) and $\mathrm{A} \beta_{1-42}$ were combined, the AUC was increased to 0.91 (95\% CI [0.86-0.95], $P<.001)$. For the adjusted (as above) $\mathrm{A} \beta_{1-42} / \mathrm{A} \beta_{1-40}$ ratios alone, the AUC was 0.79 (95\% CI [0.71-0.88], $P<.001)$, and when combined with the neuropsychological measures, AUC was 0.91 (95\%CI [0.87-0.96], $P<.001)$. The various ROC curves are displayed in Figure 1. Optimal sensitivities with specificity of at least $80 \%$ predicted probabilities are shown in Table 2. The highest sensitivity and specificity was achieved using a combination of cognitive scores and $\mathrm{A} \beta_{1-42} / \mathrm{A} \beta_{1-40}$ ratio, but this finding was driven by $\mathrm{A} \beta_{1-42}$. 
TABLE 2: Optimal sensitivities with specificities at least $80 \%$ for the various models*.

\begin{tabular}{lcccc}
\hline Model & Sensitivity & Specificity & ${ }^{\ll} \mathrm{R}^{2}$ & $\triangleleft$ Goodness-of-fit test \\
\hline Neuropsychological tests ${ }^{\dagger}$ & $67.9 \%$ & $80.0 \%$ & 0.32 & 9.32 \\
$\mathrm{~A} \beta_{1-40}$ and $\mathrm{A} \beta_{1-42}$ & $55.6 \%$ & $80.0 \%$ & 0.22 & 12.81 \\
$\mathrm{~A} \beta_{1-42} / \mathrm{A} \beta_{1-40}$ ratio & $59.3 \%$ & $80.0 \%$ & 0.22 & 6.28 \\
Neuropsychological tests and $\mathrm{A} \beta_{1-40}$ and $\mathrm{A} \beta_{1-42}$ & $85.2 \%$ & $85.9 \%$ & 0.47 & 2.31 \\
Neuropsychological tests and $\mathrm{A} \beta_{1-42} / \mathrm{A} \beta_{1-40}$ ratio & $85.2 \%$ & $86.5 \%$ & 0.49 & 4.48 \\
\hline
\end{tabular}

${ }^{*}$ Calculations based on predicted probabilities form Logistic Regression.

${ }^{\dagger}$ Modified Mini-Mental State Examination, Hopkins Verbal Learning Test-Revised Trial 4, supermarket fluency.

«Represents Negelkerke $\mathrm{R}^{2}$.

$\triangleleft^{\triangleleft}$ Hosmer and Lemeshow chi-square test of goodness of fit, a $P$ value of $>.05$ was noted and indicates that the model adequately fits the data.

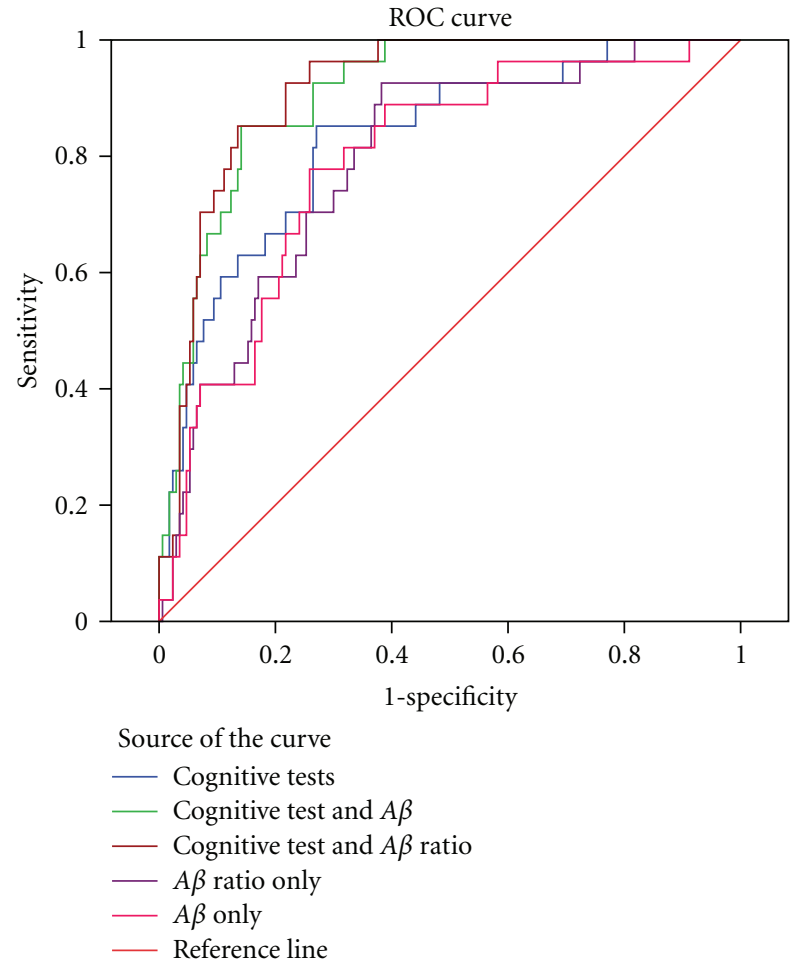

FIGURE 1

\section{Discussion}

The pathogenesis of $\mathrm{AD}$ is initiated before the clinical symptoms of cognitive impairment and functional decline become apparent in its victims. A simple and pragmatic method for identifying older adults at an increased risk for $\mathrm{MCI} / \mathrm{AD}$ who may benefit from targeted prevention is therefore of importance in reducing the burden of $\mathrm{AD}$. The combination of brief neuropsychological tests along with blood-based biomarkers of $\mathrm{AD}$ represents a reasonable approach with a potential for wide-scale use. Our findings here provide support for this notion and demonstrate that early prediction of risk for developing MCI/AD may be feasible via a combination of brief neuropsychological tests and biomarkers in an at-risk cohort. In this subcohort from ADAPT, measures of global cognitive function (3MS), episodic memory (HVLT-R Trial 4), language fluency, and serum $\mathrm{A} \beta_{1-42} / \mathrm{A} \beta_{1-40}$ ratio achieved an excellent accuracy of $91 \%$. Furthermore, sensitivity with specificity of at least $80 \%$ for the combined measures was superior to neuropsychological measures or to serum $\mathrm{A} \beta$ levels alone.

We have recently shown that $\mathrm{A} \beta$ levels alone can predict $\mathrm{MCI} / \mathrm{AD}[14]$, but $\mathrm{A} \beta$ levels are influenced by vascular disease and associated medications [44] and require adjustment to observe the full impact of $\mathrm{A} \beta$ in predictive modeling. We have also shown that in subjects diagnosed with $\mathrm{AD}$, there is an association between measures of language tests of fluency and object naming and $A \beta_{1-40}$ and that memory performance is associated with serum $A \beta_{1-42}[31]$. An association between serum $\mathrm{A} \beta_{1-40}$ and cognitive measures of memory and language has also been reported in cognitively normal older adults [46]. High baseline $\mathrm{A} \beta_{1-42}$ and $\mathrm{A} \beta_{1-40}$ with stable $\mathrm{A} \beta_{1-42}$ over time is shown to be associated with diminishing cognition [47]. More recently, Yaffe and colleagues demonstrated that low $\mathrm{A} \beta_{1-42} / \mathrm{A} \beta_{1-40}$ ratios predict cognitive decline over 9 years [48]. In our study, we demonstrate that low $\mathrm{A} \beta_{1-42}$ and $\mathrm{A} \beta_{1-42} / \mathrm{A} \beta_{1-40}$ ratios are associated with cognitive decline even within one year. This is extremely valuable from the clinical perspective, as the ability to identify at-risk individuals within a year prior to the onset can significantly improve the quality of care and the recruitment strategy for prevention trials by redirecting those individuals who may not benefit from preventive therapies towards more suitable clinical intervention. This is demonstrated by recent ADAPT findings, which suggest that individuals with low baseline cognitive scores converted soon after the trial initiated and that neither naproxen nor celecoxib intervention was beneficial to these individuals [49]. Collectively, these findings suggest that combining cognitive tests with blood $\mathrm{A} \beta$ may be useful for predicting future $\mathrm{MCI} / \mathrm{AD}$, which to date has not been explored, particularly as either $A \beta$ or the cognitive tests alone may not have the desired sensitivity or specificity for prediction of future $\mathrm{MCI} / \mathrm{AD}$.

This current work presented here provides evidence that the combination of brief neuropsychological tests and blood $\mathrm{A} \beta$ has potential utility in predicting $\mathrm{MCI} / \mathrm{AD}$ at least 2 to 4 years prior to the clinical classification of MCI or diagnosis of AD. In addition, our findings also demonstrate the importance of accounting for factors such as APOE, vascular risk factors, and medications when using $\mathrm{A} \beta$ in predicting MCI/AD. Although at present no studies 
have reported sensitivity and specificity of CSF $A \beta_{1-42}$ in predicting $\mathrm{MCI} / \mathrm{AD}$ conversion from normal cognition, a large multicenter study has shown that CSF $A \beta_{1-42}$ predicts transition from MCI to $\mathrm{AD}$ [50], while tau alone achieved a high sensitivity $(83 \%)$ with acceptable specificity $(72 \%)$. It is interesting to note that our findings using blood and cognitive tests, a far less invasive method, resulted in higher sensitivities and specificities for predicting cognitive decline in at-risk cognitively normal older adults. Despite the limitation that blood sampling was not conducted at the same time point as the cognitive testing, our data provide strong support for further evaluation of this approach, particularly as we have not seen significant fluctuations in $\mathrm{A} \beta$ levels over a one-year period (pers. Comm.).

\section{Conclusion}

Our study provides support that blood-based $\mathrm{A} \beta$ levels may have diagnostic utility when combined with neuropsychological measures. This proposed method warrants further investigation to determine its practical applicability in specialized clinic setting by allied health personal and in routine primary care clinics.

\section{Disclosure}

The authors report no conflict of interests.

\section{Acknowledgments}

The ADAPT study was supported by the National Institute of Aging through the National Institute of Health Grant no. NIH 7U01AG15477-02. Portions of this study were also supported by the Alzheimer's Association (NIRG-09131751) and through the generous support from the Robert and Diane Roskamp Foundation.

\section{References}

[1] R. Brookmeyer, E. Johnson, K. Ziegler-Graham, and H. M. Arrighi, "Forecasting the global burden of Alzheimer's disease," Alzheimer's and Dementia, vol. 3, no. 3, pp. 186-191, 2007.

[2] D. L. Weimer and M. A. Sager, "Early identification and treatment of Alzheimer's disease: social and fiscal outcomes," Alzheimer's and Dementia, vol. 5, no. 3, pp. 215-226, 2009.

[3] C. Holmes, D. Boche, D. Wilkinson et al., "Long-term effects of Abeta42 immunisation in Alzheimer's disease: follow-up of a randomized, placebo-controlled phase 1 trial," Lancet, vol. 372, pp. 216-223, 2008.

[4] D. Blacker, H. Lee, A. Muzikansky et al., "Neuropsychological measures in normal individuals that predict subsequent cognitive decline," Archives of Neurology, vol. 64, no. 6, pp. 862-871, 2007.

[5] M. W. Bondi, D. P. Salmon, D. Galasko, R. G. Thomas, and L. J. Thal, "Neuropsychological function and apolipoprotein E genotype in the preclinical detection of Alzheimer's disease," Psychology and Aging, vol. 14, no. 2, pp. 295-303, 1999.

[6] H. Amieva, M. Le Goff, X. Millet et al., "Prodromal Alzheimer's disease: successive emergence of the clinical symptoms," Annals of Neurology, vol. 64, no. 5, pp. 492-498, 2008.

[7] G. W. Small, A. La Rue, S. Komo, A. Kaplan, and M. A. Mandelkern, "Predictors of cognitive change in middle-aged and older adults with memory loss," American Journal of Psychiatry, vol. 152, no. 12, pp. 1757-1764, 1995.

[8] W. E. Klunk, H. Engler, A. Nordberg et al., "Imaging brain amyloid in Alzheimer's disease with pittsburgh compound-B," Annals of Neurology, vol. 55, no. 3, pp. 309-319, 2004.

[9] G. W. Small, V. Kepe, L. M. Ercoli et al., "PET of brain amyloid and tau in mild cognitive impairment," New England Journal of Medicine, vol. 355, no. 25, pp. 2652-2663, 2006.

[10] P. Vemuri, H. J. Wiste, S. D. Weigand et al., "MRI and CSF biomarkers in normal, $\mathrm{MCI}$, and $\mathrm{AD}$ subjects: predicting future clinical change," Neurology, vol. 73, no. 4, pp. 294-301, 2009.

[11] A. M. Fagan, C. M. Roe, C. Xiong, M. A. Mintun, J. C. Morris, and D. M. Holtzman, "Cerebrospinal fluid tau/ $\beta$-amyloid (42) ratio as a prediction of cognitive decline in nondemented older adults," Archives of Neurology, vol. 64, no. 3, pp. 343-349, 2007.

[12] N. R. Graff-Radford, J. E. Crook, J. Lucas et al., "Association of low plasma $\mathrm{A} \beta 42 / \mathrm{A} \beta 40$ ratios with increased imminent risk for mild cognitive impairment and Alzheimer' disease," Archives of Neurology, vol. 64, no. 3, pp. 354-362, 2007.

[13] O. I. Okereke, W. Xia, D. J. Selkoe, and F. Grodstein, “Tenyear change in plasma amyloid $\beta$ levels and late-life cognitive decline," Archives of Neurology, vol. 66, no. 10, pp. 1247-1253, 2009.

[14] L. Abdullah, C. Luis, D. Paris et al., "Serum A $\beta$ levels as predictors of conversion to mild cognitive impairment/Alzheimer disease in an ADAPT subcohort," Molecular Medicine, vol. 15, no. 11-12, pp. 432-437, 2009.

[15] R. J. Bateman, G. Wen, J. C. Morris, and D. M. Holtzman, "Fluctuations of CSF amyloid- $\beta$ levels: implications for a diagnostic and therapeutic biomarker," Neurology, vol. 68, no. 9, pp. 666-669, 2007.

[16] L. Abdullah, D. Paris, C. Luis et al., "The influence of diagnosis, intra- and inter-person variability on serum and plasma A $\beta$ levels," Neuroscience Letters, vol. 428, no. 2-3, pp. 53-58, 2007.

[17] D. R. Lachno, H. Vanderstichele, G. De Groote et al., "The influence of matrix type, diurnal rhythm and sample collection and processing on the measurement of plasma betaamyloid isoforms using the INNO-BIA plasma Abeta forms multiplex assay," The Journal of Nutrition, Health and Aging, vol. 13, pp. 220-225, 2009.

[18] N. Ertekin-Taner, L. H. Younkin, D. M. Yager et al., "Plasma amyloid $\beta$ protein is elevated in late-onset Alzheimer' disease families," Neurology, vol. 70, no. 8, pp. 596-606, 2008.

[19] P. W. Thompson and A. Lockhart, "Monitoring the amyloid beta-peptide in vivo-caveat emptor," Drug Discovery Today, vol. 14, no. 5-6, pp. 241-251, 2009.

[20] C. Meinert, L. McCaffrey, and J. Breitner, “Alzheimer's disease anti-inflammatory prevention trial: design, methods and baseline results," Alzheimer's Dement, vol. 5, pp. 93-104, 2009.

[21] E. L. Teng and H. C. Chui, "The modified mini-mental state (MMS) examination," Journal of Clinical Psychiatry, vol. 48, no. 8, pp. 314-318, 1987.

[22] J. Brandt and R. Benedict, "The Hopkins verbal learning test: development of a new memory test with six equivalent forms," Clinical Neuropsychologist, vol. 5, no. 2, pp. 125-142, 1991.

[23] D. Wechsler, Wechsler Adult Intelligence Scale-Revised. Manual, Psychological Corporation, New York, NY, USA, 1981. 
[24] B. Wilson, J. Cockburn, A. Baddeley, and R. Hiorns, "The development and validation of a test battery for detecting and monitoring everyday memory problems," Journal of Clinical and Experimental Neuropsychology, vol. 11, no. 6, pp. 855-870, 1989.

[25] R. Benedict, C. Schrectlen, L. Groninger et al., "Revision of the brief visual spatial memory tests: studies of normal performance, reliability and validity," Psychological Assessment, vol. 10, pp. 31-39, 1996.

[26] M. F. Folstein, S. E. Folstein, and P. R. McHugh, "Mini mental state. A practical method for grading the cognitive state of patients for the clinician," Journal of Psychiatric Research, vol. 12, no. 3, pp. 189-198, 1975.

[27] J. Yesavage, T. Brink, T. Rose et al., "Development and validation of a geriatric depression screening scale: a preliminary report," Journal of Psychiatric Research, vol. 17, pp. 37-49, 1983.

[28] L. Squire, C. Wetzel, and P. Slater, "Memory complaints after electroconvulsive therapy: assessment with a new self-rating scale instrument," Biological Psychiatry, vol. 14, pp. 791-801, 1979.

[29] C. M. Clark and D. C. Ewbank, "Performance of the dementia severity rating scale: a caregiver questionnaire for rating severity in Alzheimer' disease," Alzheimer Disease and Associated Disorders, vol. 10, no. 1, pp. 31-39, 1996.

[30] C. A. de Jager, A. C. M. C. Schrijnemaekers, T. E. M. Honey, and M. M. Budge, "Detection of MCI in the clinic: evaluation of the sensitivity and specificity of a computerised test battery, the Hopkins verbal learning test and the MMSE," Age and Ageing, vol. 38, no. 4, pp. 455-460, 2009.

[31] C. A. Luis, L. Abdullah, D. Paris et al., "Serum $\beta$-amyloid correlates with neuropsychological impairment," Aging, Neuropsychology, and Cognition, vol. 16, no. 2, pp. 203-218, 2009.

[32] J. T. Tschanz, K. A. Welsh-Bohmer, I. Skoog et al., "Dementia diagnoses from clinical and neuropsychological data compared: the cache county study," Neurology, vol. 54, no. 6, pp. 1290-1296, 2000.

[33] J. C. Morris, A. Heyman, R. C. Mohs et al., "The consortium to establish a registry for Alzheimer's disease (CERAD). Part I. Clinical and neuropsychological assessment of Alzheimer's disease," Neurology, vol. 39, no. 9, pp. 1159-1165, 1989.

[34] D. Wechsler, Wechsler Memory Scale—Revised, Psychological Corporation, San Antonio, Tex, USA, 1987.

[35] A. Benton, K. Hamsher, and A. Siven, Multilingual Aphasia Examination, AJA Associates, Iowa City, Calif, USA, 3rd edition, 1994

[36] S. W. Sumerall, P. L. Timmons, A. L. James, M. J. M. Ewing, and M. E. Oehlert, "Expanded norms for the controlled oral word association test," Journal of Clinical Psychology, vol. 53, no. 5, pp. 517-521, 1997.

[37] R. Reitan, Trail Making Test: Manual for Administering and Scoring, Reitan Neuropsychological Laboratory, Tucson, Ariz, USA, 1986.

[38] A. Smith, Symbol Digit Modalities Test-Manual, Western Psychological Services, Los Angeles, Calif, USA, 1982.

[39] R. Zachary, Shipley Institute of Living Scale-Revised, Western Psychological Services, Los Angeles, Calif, USA, 1991.

[40] G. McKhann, D. Drachman, M. Folstein et al., "Clinical diagnosis of Alzheimer's disease: report of the NINCDSADRDA work group under the auspices of department of health and human services task force on Alzheimer's disease," Neurology, vol. 34, pp. 939-944, 1984.
[41] R. C. Petersen, G. E. Smith, S. C. Waring, R. J. Ivnik, E. G. Tangalos, and E. Kokmen, "Mild cognitive impairment: clinical characterization and outcome," Archives of Neurology, vol. 56, no. 3, pp. 303-308, 1999.

[42] R. C. Petersen, R. Doody, A. Kurz et al., "Current concepts in mild cognitive impairment," Archives of Neurology, vol. 58, no. 12, pp. 1985-1992, 2001.

[43] J. L. Whitwell, R. C. Petersen, S. Negash et al., "Patterns of atrophy differ among specific subtypes of mild cognitive impairment," Archives of Neurology, vol. 64, no. 8, pp. 11301138, 2007.

[44] L. Abdullah, C. Luis, D. Paris et al., "High serum A $\beta$ and vascular risk factors in first-degree relatives of Alzheimer's disease patients," Molecular Medicine, vol. 15, no. 3-4, pp. 95100, 2009.

[45] C. G. Lyketsos, J. C. S. Breitner, R. C. Green et al., "Naproxen and celecoxib do not prevent $\mathrm{AD}$ in early results from a randomized clinical trial," Neurology, vol. 68, no. 21, pp. 1800$1808,2007$.

[46] J. Gunstad, M. B. Spitznagel, E. Glickman et al., “ $\beta$-amyloid is associated with reduced cognitive function in healthy older adults," Journal of Neuropsychiatry and Clinical Neurosciences, vol. 20, no. 3, pp. 327-330, 2008.

[47] S. Cosention, Y. Stern, E. Sokolov et al., "Plasma beta-amyloid and cognitive decline," Archives of Neurology. In press.

[48] K. Yaffe, A. Weston, N. R. Graff-Radford et al., "Association of plasma beta-amyloid level and cognitive reserve with subsequent cognitive decline," Journal of the American Medical Association, vol. 305, pp. 261-266, 2011.

[49] J. C. S. Breitner, "Onset of Alzheimer's dementia occurs commonly without prior cognitive impairment: results from the Alzheimer's disease anti-inflammatory prevention trial (ADAPT) ," Alzheimers Dement, vol. 4, no. 4, supplement 1, pp. T130-T131, 2008.

[50] N. Mattsson, H. Zetterberg, O. Hansson et al., "CSF biomarkers and incipient Alzheimer' disease in patients with mild cognitive impairment," Journal of the American Medical Association, vol. 302, no. 4, pp. 385-393, 2009. 


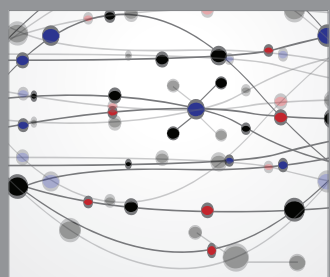

The Scientific World Journal
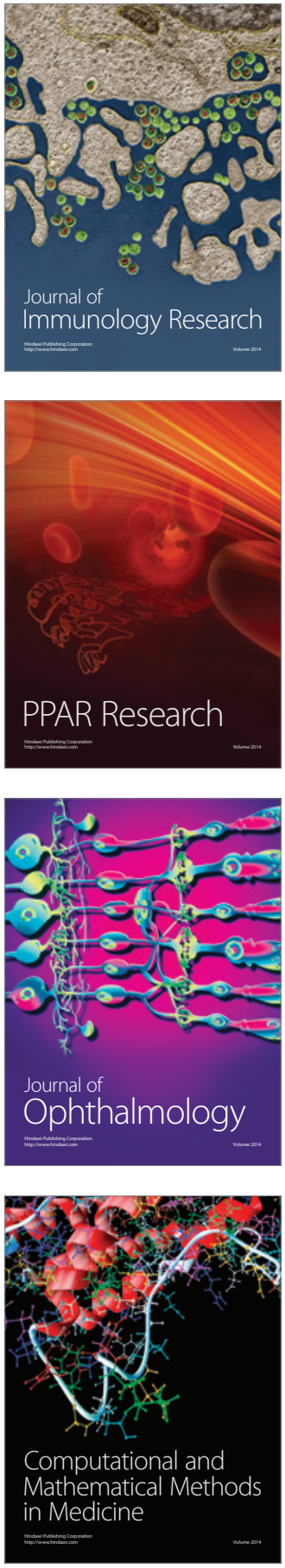

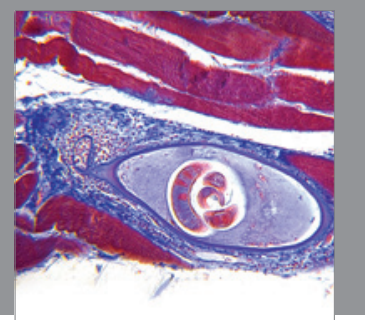

Gastroenterology

Research and Practice
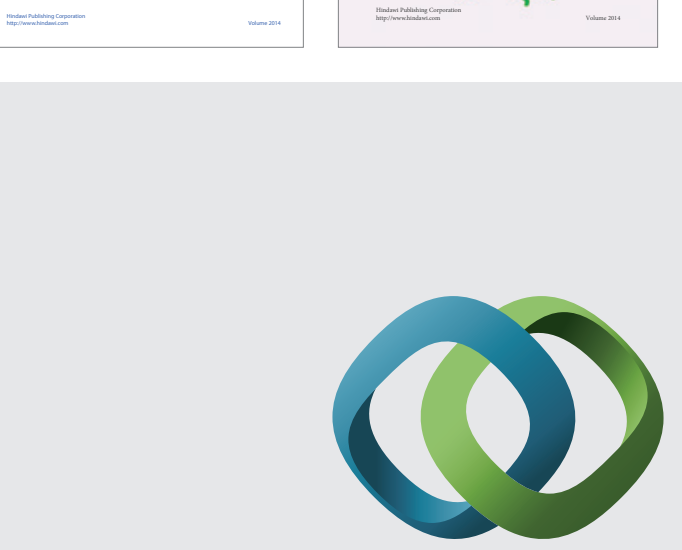

\section{Hindawi}

Submit your manuscripts at

http://www.hindawi.com
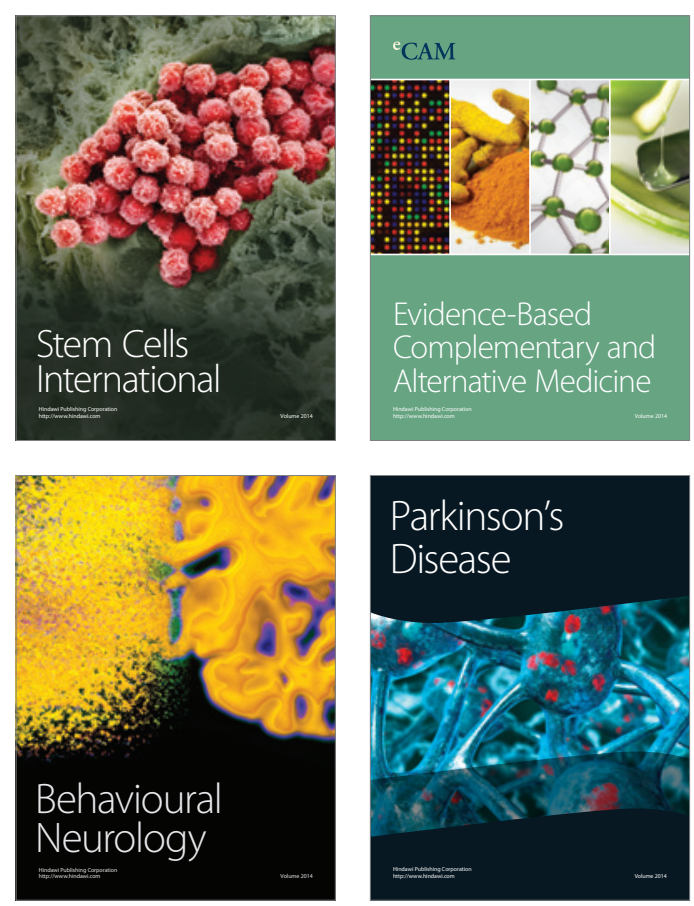

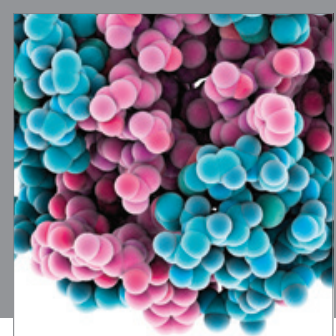

Journal of
Diabetes Research

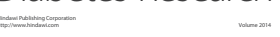

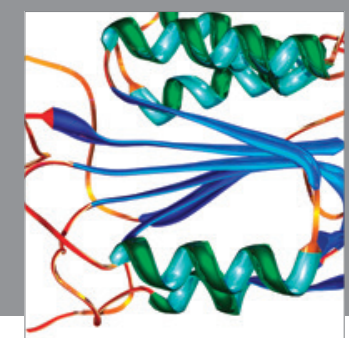

Disease Markers
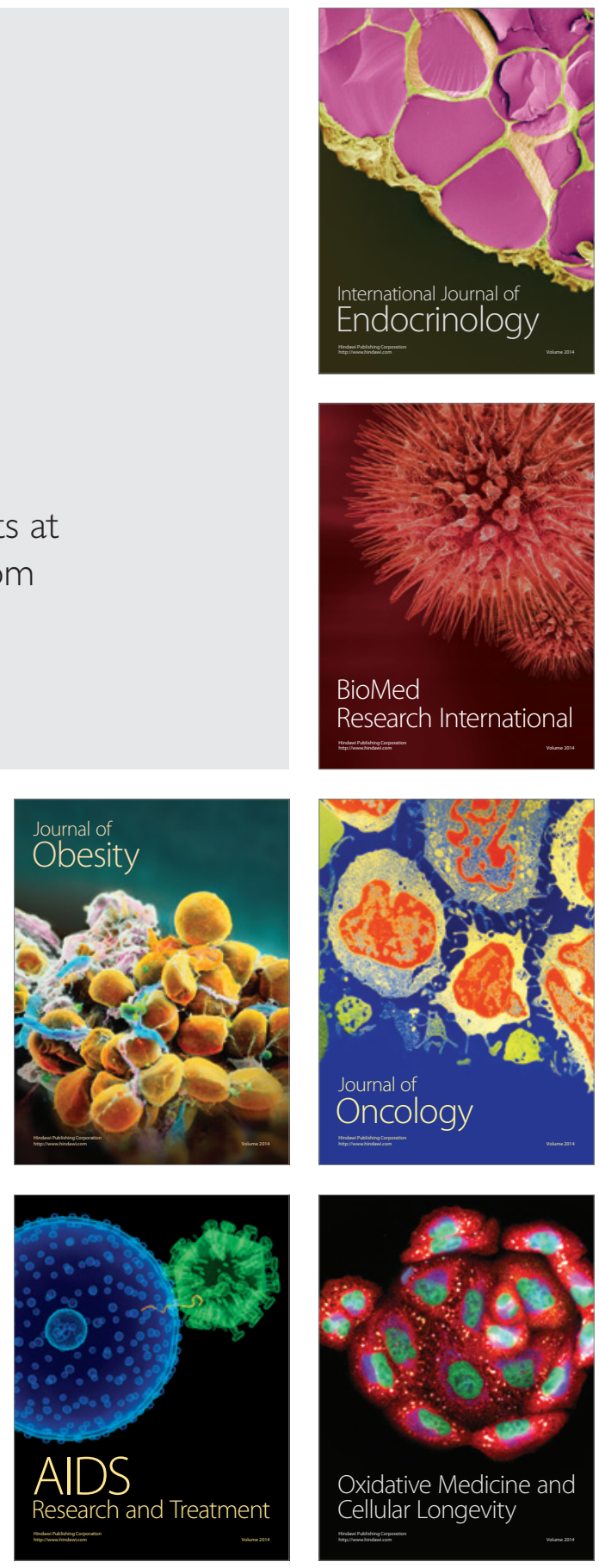